\title{
News of Cognitive Cure for Age-Related Brain Shrinkage Is Premature: A Comment on Burgmans et al. (2009)
}

\author{
Naftali Raz \\ Wayne State University
}

\author{
Ulman Lindenberger \\ Max Planck Institute for Human Development
}

\begin{abstract}
The extant longitudinal literature consistently supports the notion of age-related declines in human brain volume. In a report on a longitudinal cognitive follow-up with cross-sectional brain measurements, Burgmans and colleagues (2009) claim that the extant studies overestimate brain volume declines, presumably due to inclusion of participants with preclinical cognitive pathology. Moreover, the authors of the article assert that such declines are absent among optimally healthy adults who maintain cognitive stability for several years. In this comment accompanied by reanalysis of previously published data, we argue that these claims are incorrect on logical, methodological, and empirical grounds.
\end{abstract}

Keywords: aging, MRI, brain, cognition, longitudinal
Multiple cross-sectional studies have demonstrated that advanced age is associated with reduced volumes of the brain parenchyma (for reviews, see Raz, 2005, and Raz \& Kennedy, 2009). However, the utility of these findings for understanding brain aging is limited because mean rates of change and individual differences in rates of change cannot be assessed within crosssectional designs (Hofer, Flaherty, \& Hoffman, 2006; Lindenberger \& Pötter, 1998). Moreover, in cross-sectional studies, age differences in attributes of interest are inexorably confounded by other individual differences associated with age (Hofer et al., 2006; Lindenberger \& Pötter, 1998). For instance, cross-sectional samples of ostensibly healthy individuals may include undetected preclinical cases of dementia (Sliwinski \& Buschke, 1999), and the prevalence of such cases may increase with age.

Longitudinal studies, which are almost entirely free of the abovementioned problems, show that most of the cortical regions and subcortical structures indeed shrink with the passage of time (reviewed in Raz, 2005; Raz \& Kennedy, 2009). Moreover, at least for some regions, the magnitude of longitudinal change exceeds cross-sectional estimates (Raz et al., 2005), thus implying that cross-sectional studies underestimate the extent of regional brain shrinkage.

The rate of age-related shrinkage varies reliably among individuals and across brain regions (Raz \& Kennedy, 2009; Raz et al., 2005). Vascular risk factors, such as hypertension and diabetes,

Naftali Raz, Department of Psychology and Institute of Gerontology, Wayne State University; Ulman Lindenberger, Max Planck Institute for Human Development, Berlin, Germany.

This research was supported in part by National Institute on Aging Grant R37 AG-011230 to Naftali Raz. This article was written while Ulman Lindenberger was a fellow at the Center for Advanced Study in the Behavioral Sciences at Stanford University.

The author of the commented article, Saartje Burgmans, was a visiting student-scholar in Naftali Raz's laboratory and currently collaborates with Naftali Raz on a project unrelated to the commented article. Ulman Lindenberger has no declared conflicts.

Correspondence concerning this article should be addressed to Naftali Raz, 87 East Ferry Street, Detroit MI 48202. E-mail: nraz@wayne.edu exacerbate the declines (e.g., Resnick, Pham, Kraut, Zonderman, \& Davatzikos, 2003; Raz, Rodrigue, \& Acker, 2003; Raz et al., 2005; Raz, Rodrigue, Kennedy, \& Acker, 2007). Accelerated reduction in regional (medial temporal) volumes precedes the onset of cognitive pathology (Jack et al., 2000; De Toledo-Morrell et al., 2004). Even in healthy persons, shrinkage of the entorhinal cortex has been linked to poor memory performance (Rodrigue \& Raz, 2004) and lower fluid intelligence (Raz, Lindenberger, Ghisletta, Rodrigue, Kennedy, \& Acker, 2008).

Burgmans and colleagues (2009) report that in individuals selected for the lack of reliable cognitive decline, there are no reliable negative associations between age and brain volume. This finding is not surprising, and it does not help to elucidate the relation between cognitive decline and brain shrinkage. Nevertheless, Burgmans and colleagues conclude, on the basis of their results, that the extant studies overestimate brain volume declines. Here, we show that this conclusion is unwarranted on logical, methodological, and empirical grounds.

The argument offered by Burgmans and colleagues (2009) takes the following form: (a) If people show cognitive decline, then their brain volumes shrink. (b) Some people show no cognitive decline. (c) Therefore, the brains of these people do not shrink. However, whether people without cognitive decline may or may not show brain shrinkage (a question addressed empirically below) cannot be derived from the observation that they show no cognitive decline. Making such an inference would correspond to denying the antecedent, a logical fallacy. In other words, examining individuals who do not decline cognitively renders the search for causes of cognitive decline, such as brain volume shrinkage, curiously difficult.

Although it may be unclear from its title, the Burgmans et al. (2009) study is not a longitudinal investigation with regard to the brain volume measures. Thus, their study offers no basis for observing brain "atrophy," or "change." Without repeated MRI assessment, one can claim only that persons who evidenced cognitive decline had smaller regional volumes and that older decliners had even smaller volumes than their younger counterparts, and in the cognitively stable group, age differences were not reliable. Thus, the longitudinal conclusions drawn by Burgmans et al. are not warranted by their analyses of the cross-sectional data. 
Burgmans and colleagues (2009) infer that "the age effect in previous studies may partly have been caused by the inclusion of participants with subclinical cognitive disorders" (p. 547). This conjecture is likely to be correct for cross-sectional investigations (see Sliwinski \& Buschke, 1999, regarding this problem in cognitive studies), but it is much less relevant to longitudinal studies. When participants undergo repeated assessment of brain and cognition (e.g., Raz et al., 2005, 2007, 2008), cases with cognitive disorders can be more easily identified and removed from the sample than in cross-sectional studies so that the contamination of findings by preclinical cases is less likely.

The question of whether a small and selected group of individuals who are free of disease and health risks and who maintain stability on age-sensitive cognitive measures over a significant period of time escapes brain shrinkages can be addressed empirically, but the answer cannot emerge from a study that lacks the required longitudinal evidence on brain volume shrinkage. To test this hypothesis, we reanalyzed the data previously reported in Raz et al. (2008). In a sample of 87 adults, who participated in that study, 55 persons were free of manifest vascular disease, including hypertension, at baseline and at 5-year follow-up. In that optimally healthy group, 40 participants evidenced declining scores on Cattell IQ, a measure of fluid reasoning that is highly sensitive to aging, whereas 15 did not. When we restricted the age of the sample at 49 and above, as in Burgmans et al. (2009), there were 10 cognitively "stable" individuals and 20 "decliners." The cognitive change factors (decliners vs. stable) were entered in the general linear model as a grouping factor. The model also included age (centered at the sample mean) as a continuous predictor, time (baseline vs. follow-up), and region of interest (ROI) as repeated measures factors.

The analyses of that subsample revealed no age differences in regional brain volumes, $F(1,27)=1.16, p=.29$. Cognitively stable participants did not differ reliably in ROI volumes from cognitive decliners, $F(1,27)=1.39, p=.25$. A nonsignificant Age $\times$ Cognitive Change interaction $(F<1)$ was removed from the model. We found a robust time effect, $F(1,27)=12.059, p=.002$. There also was a marginally significant Time $\times$ ROI interaction, $F(1$, $27)=4.00, p=.05$, but no Time $\times$ Age or Time $\times$ Cognitive Change interactions $(F \mathrm{~s}<1)$. The latter two results are most pertinent to the claim articulated by Burgmans and colleagues (2009). Our findings indicate that even in a sample of optimally healthy middleaged and older adults, at least some brain regions reliably shrink over time, suggesting that cognitive stability provides no protection against shrinkage. Note that shrinkage was reliably different from zero despite the small sample size, documenting the superior statistical power of longitudinal comparisons.

We hasten to add that the separation of a relatively homogeneous healthy sample into stable and declining individuals is in itself problematic, especially if a theory positing that the groups of stable and declining individuals represent different etiological entities is lacking. First, reliability for any behavioral assessment is less than perfect, and the effects of unreliability are magnified when differences are computed on observed scores. Second, whether an observed longitudinal difference reflects reliable declines depends on the degree of day-to-day fluctuations in cognitive performance (e.g., Nesselroade, 1991), which may vary from person to person and are not assessed in most longitudinal investigations. Consequently, the error rates in classifying people into groups of stable and declining individuals are not known, and the validity of such classification is unclear. Therefore, it is more sensible to regard the magnitude and rate of decline as continuously distributed parameters and specify them as variance terms, or random effects, in statistical analysis.

In their critique of previous reports, Burgmans et al. (2009) allude to a possibility that findings of brain shrinkage stem from studies that employed insufficiently rigorous screening of participants. This allusion contradicts the facts. The extant longitudinal studies that report regional brain shrinkage applied more stringent screening criteria than Burgmans et al., including screening of participants with Mini-Mental State Examination scores below 26, and a history of hypertension, diabetes, minor head injury, and nonpsychotic depressive symptomatology (e.g., Raz et al., 2005).

In summary, neither the extant literature, nor logical analysis of presented inference, nor the results of an empirical test support the hypothesis that the extent of brain shrinkage is overestimated. Although the mechanisms of regional brain shrinkage are unclear, it is a reliably observed phenomenon. Maintaining the level of cognitive performance throughout late adulthood may convey multiple benefits, and may even reduce the negative effects of aging on everyday competence. However, available evidence does not support the contention that cognitive fitness can stop age-related brain decline. Cognitive and motor training can sometimes lead to transient local alterations of MRI signal that are interpreted as gray matter increases (e.g., Draganski et al., 2006). The neural mechanisms of such alterations are unknown, and whether they can result in sustainable attenuation of brain shrinkage is unclear. As it stands, the assertion that "as long as people stay cognitively healthy, there may be no substantial gray matter atrophy in several brain areas that are highly associated with cognition" (p. 547) is unwarranted on logical, methodological, and empirical grounds.

\section{References}

Burgmans, S., van Boxtel, M. P., Vuurman, E. F., Smeets, F., Gronenschild, E. H., Uylings, H. B., \& Jolles, J. (2009). The prevalence of cortical gray matter atrophy may be overestimated in the healthy aging brain. Neuropsychology, 23, 541-550.

De Toledo-Morrell, L., Stoub, T. R., Bulgakova, M., Wilson, R. S., Bennett, D. A., Leurgans, S., ... Turner, D. A. (2004). MRI-derived entorhinal volume is a good predictor of conversion from MCI to AD. Neurobiology of Aging, 25, 1197-1203.

Draganski, B., Gaser, C., Kempermann, G., Kuhn, H. G., Winkler, J., Büchel, C., \& May, A. (2006). Temporal and spatial dynamics of brain structure changes during extensive learning. Journal of Neuroscience, 26, 6314-6317.

Hofer, S. M., Flaherty, B. P., \& Hoffman, L. (2006). Cross-sectional analysis of time-dependent data: Mean-induced association in age-heterogeneous samples and an alternative method based on sequential narrow age-cohort samples. Multivariate Behavioral Research, 41, 165-187.

Jack, C. R., Jr., Petersen, R. C., Xu, Y., O’Brien, P. C., Smith, G. E., Ivnik, R. J., .. Kokmen, E. (2000). Rates of hippocampal atrophy correlate with change in clinical status in aging and AD. Neurology, 55, 484-489.

Lindenberger, U., \& Pötter, U. (1998). The complex nature of unique and shared effects in hierarchical linear regression: Implications for developmental psychology. Psychological Methods, 3, 218-230.

Nesselroade, J. R. (1991). The warp and woof of the developmental fabric. In R. Downs, L. Liben, \& D. Palermo (Eds.), Visions of development, the environment, and aesthetics: The legacy of Joachim F. Wohlwill (pp. 213-240). Hillsdale, NJ: Erlbaum. 
Raz, N. (2005). The aging brain observed in vivo: Differential changes and their modifiers. In R. Cabeza, L. Nyberg, \& D. Park (Eds.), Cognitive neuroscience of aging: Linking cognitive and cerebral aging (pp. 1755). New York: Oxford University Press.

Raz, N., Gunning-Dixon, F., Head, D., Williamson, A., Rodrigue, K., \& Acker, J. D. (2004). Aging, sexual dimorphism, and hemispheric asymmetry of the cerebral cortex: Replicability of regional differences in volume. Neurobiology of Aging, 25, 377-396.

Raz, N., \& Kennedy, K. M. (2009). A systems approach to age-related change: Neuroanatomical changes, their modifiers, and cognitive correlates. In W. Jagust \& M. D'Esposito (Eds.), Imaging the aging brain (pp. 43-70). New York: Oxford University Press.

Raz, N., Lindenberger, U., Ghisletta, P., Rodrigue, K. M., Kennedy, K. M., \& Acker, J. D. (2008). Neuroanatomical correlates of fluid intelligence in healthy adults and persons with vascular risk factors. Cerebral Cortex, 18, 718-726.

Raz, N., Lindenberger, U., Rodrigue, K. M., Kennedy, K. M., Head, D., Williamson, A., . . Acker, J. D. (2005). Regional brain changes in aging healthy adults: General trends, individual differences and modifiers. Cerebral Cortex, 15, 1676-1689.
Raz, N., Rodrigue, K. M., \& Acker, J. D. (2003). Hypertension and the brain: Vulnerability of the prefrontal regions and executive functions. Behavioral Neuroscience, 117, 1169-1180.

Raz, N., Rodrigue, K. M., Kennedy, K. M., \& Acker, J. D. (2007). Vascular health and longitudinal changes in brain and cognition in middle-aged and older adults. Neuropsychology, 212, 149-157.

Resnick, S. M., Pham, D. L., Kraut, M. A., Zonderman, A. B., \& Davatzikos, C. (2003). Longitudinal magnetic resonance imaging studies of older adults: A shrinking brain. Journal of Neuroscience, 23, 3295-3301.

Rodrigue, K. M., \& Raz, N. (2004). Shrinkage of the entorhinal cortex over five years predicts memory performance in healthy adults. Journal of Neuroscience, 24, 956-963.

Sliwinski, M., \& Buschke, H. (1999). Cross-sectional and longitudinal relationships among age, cognition, and processing speed. Psychology of Aging, 14, 18-33.

Received September 17, 2009

Revision received November 25, 2009

Accepted December 8, 2009

\section{Call for Papers: Journal of Experimental Psychology: Learning, Memory, and Cognition Special Section on Neural Mechanisms of Analogical Reasoning}

The Journal of Experimental Psychology: Learning, Memory, and Cognition invites submissions of manuscripts for a special section on the Neural Mechanisms of Analogical Reasoning to be compiled by Associate Editor Miriam Bassok and Guest Editors Kevin Dunbar and Keith Holyoak. The goal of the special section is to showcase high-quality research that brings together behavioral, neuropsychological, computational, and neuroimaging approaches to understanding the cognitive and neural mechanisms that are involved in analogical reasoning. The editors are seeking articles on analogy and related cognitive processes (e.g., schema induction, metaphor, role-based relational reasoning, category-based induction) that either present original research using methods of cognitive neuroscience or that present behavioral research (including studies of cognitive development and/or aging and studies of brain-damaged patients) strongly connected to the neural mechanisms of analogical reasoning.

The submission deadline is October 1, 2010. The main text of each manuscript, exclusive of figures, tables, references, or appendixes, should not exceed 35 double-spaced pages (approximately 7,500 words). Initial inquiries regarding the special section may be sent to Miriam Bassok (mbassoku.washington.edu). Papers should be submitted through the journal's submission portal (http://www.apa.org/pubs/journals/xlm) with a cover letter indicating that the paper is to be considered for the special section. 